\title{
First Observation of (Anti)Deuterons in DIS
}

\author{
S. Chekanov \\ For the ZEUS Collaboration \\ DESY Laboratory, 22607, Hamburg, Germany. \\ On leave from the HEP division, Argonne National Laboratory, \\ 9700 S.Cass Avenue, Argonne, IL 60439, USA \\ E-mail: chekanov@mail.desy.de
}

\begin{abstract}
First observation of (anti)deuterons in deep inelastic ep scattering (DIS) measured with the ZEUS detector at HERA is reported. The production rate of deuterons is higher than that of antideuterons. However, no asymmetry in the production rate of protons and antiprotons was found. The (anti)deuteron yield is approximately three orders of magnitude smaller than that of (anti)protons, which is consistent with the world measurements.
\end{abstract}

\section{Introduction}

Deuterons $(d)$ are loosely bound states whose production in high energy collisions can hardly be accommodated in the standard fragmentation models. The measurements of such particles may provide an important information on the structure of fragmentation region [2] and on the formation of multiquark states [3].

In collisions involving elementary particles, several measurements have been performed in order to understand the production of $d(\bar{d})$. The cross section of $\bar{d}$ in $e^{+} e^{-} \rightarrow q \bar{q}$ collisions [4] is lower than that measured in hadronic $\Upsilon(1 S)$ and $\Upsilon(2 S)$ decays, and disagrees with the predictions based on the LUND string model [5]. The $(\bar{d})$ rate in $e^{+} e^{-} \rightarrow q \bar{q}$ is also lower than that in hadronic ( $p A[6], p p[7])$ and in $\gamma p$ collisions at HERA [8], but higher than that in nucleus-nucleus $(A A)$ collisions [9].

According to the coalescence model [2] developed for heavy-ion collisions, the $d$ rate is determined by the overlap between the wave function of a proton, $p$, and a neutron, $n$, with the wave function of a $d$. The $d$ cross section is the product of single-particle cross sections for $p$ and $n$, with a coefficient of proportionality, $B_{2}$, reflecting the spatial size of the fragmentation region emitting the particles.

Unlike studies in $A A, A p$ and $p p$, all previous measurements in collisions involving elementary particles have been performed for $\bar{d}$, since the reconstruction of $d$ requires a careful separation of such states from particles produced in interactions of colliding beams with residual gas in the beam pipe and secondary interactions of particles on detector material. The deep inelastic scattering (DIS) events, which are characterised by the presence of a scattered electron in the final state, provide an ideal environment for studies of $d$, since the background from non-ep interactions is minimal. In addition, during the 1996-2000 data taking, the ZEUS detector had a small amount of inactive material between the interaction region and the central tracking detector (CTD). This material consisted of the central beam-pipe and inner wall of the CTD, with the overall thickness of $2.9 \mathrm{~mm}$ of Al. This leads to a small absorption rate of $\bar{d}$, as well as to a small contribution from secondary deuterons. 
ZEUS
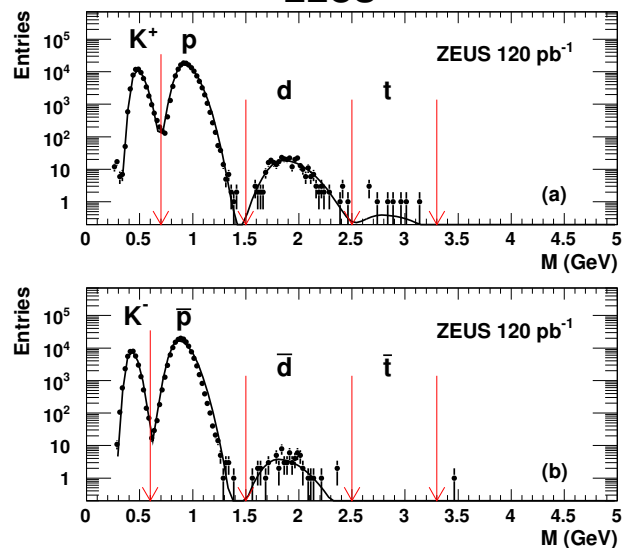

ZEUS
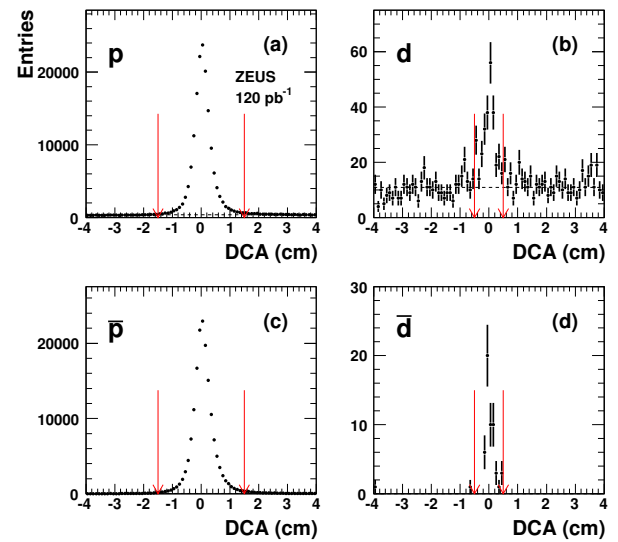

Figure 1. Left: The mass spectra reconstructed using $d E / d x$ for positive and negative particles. Right: The $D C A$ distributions for: (a)-(b) particles and (c)-(d) antiparticles.

The data sample used in the analysis corresponds to an integrated luminosity of $120 \mathrm{pb}^{-1}$ taken between 1996 and 2000 with the ZEUS detector at HERA. After a DIS selection, the average $Q^{2}$ for the data sample was about $10 \mathrm{GeV}^{2}$. The particle identification was performed using the $d E / d x$ measurement. The masses, shown in Fig. 1(left), were calculated from the measured track momentum and energy loss using the Bethe-Bloch formula.

To identify particles produced in $e p$ collisions, the distance of closest approach $(D C A)$ of the track to the beam spot in the transverse plane was used, since particles originating from the primary ep collisions feature small values of $|D C A|$. The $D C A$ distribution after the mass cuts and an additional cut on the distance, $\Delta Z$, of the $Z$-component of the track helix to the primary vertex, is shown in Fig. 1 (right). The number of particles produced in $e p$ was assessed from the $D C A$ distribution by using a side-band background subtraction. The numbers of $d$ and $\bar{d}$ after the side-band background subtraction were $177 \pm 17$ and $53 \pm 7$, respectively. This difference was found to be unlikely related to the CTD efficiency, which usually leads to a larger number of negative tracks compared to positive ones: For example, the number of reconstructed $p(\bar{p})$ in the data after the $D C A$ side-band background subtraction was $1.52 \times 10^{5}\left(1.62 \times 10^{5}\right)$. Such $p-\bar{p}$ symmetry is fully accounted for by known difference in the tracking efficiency for positive and negative tracks.

Several sources of background processes for the $d$ sample were considered [10]: events due to interactions of the incoming proton (or electron) beam with residual gas in the beam pipe (termed beam-gas interactions) and secondary interactions of particles on material between the interaction point and the central tracking detector. Extensive checks have been performed to exclude the first source. In particular, a special event selection was used for non-colliding electron and proton bunches. It was found that, after the DIS event selection, a contribution from the beam-gas events is unlikely.

Even for a clean DIS sample, $d$ can still be produced by secondary interactions of particles on inactive material. One possible source for $d$ is the reaction $N+N \rightarrow d+\pi^{+}$, where one nucleon, $N$, is produced by ep collisions, while the second one comes from inactive material in front of the CTD. Secondary $d$ can also be produced in the pickup reaction $p+n \rightarrow d$ by primary nucleon interacting in the surrounding material. Checks for such sources of 
background were either negative or not conclusive due to insufficient information on the production cross section of the pickup process. In particular, a check was done using the HERAII data collected with the ZEUS detector equipped with a vertex detector. For the $d E / d x$ measurement, this additional detector increased the overall material between the interaction point and the CTD by a factor three. As expected, the production rate of $d$ has significantly increased due to a stronger contribution from spallation processes. However, the rate of $\bar{d}$ after the $D C A$ background subtraction was the same as for HERAI.

\section{ZEUS}
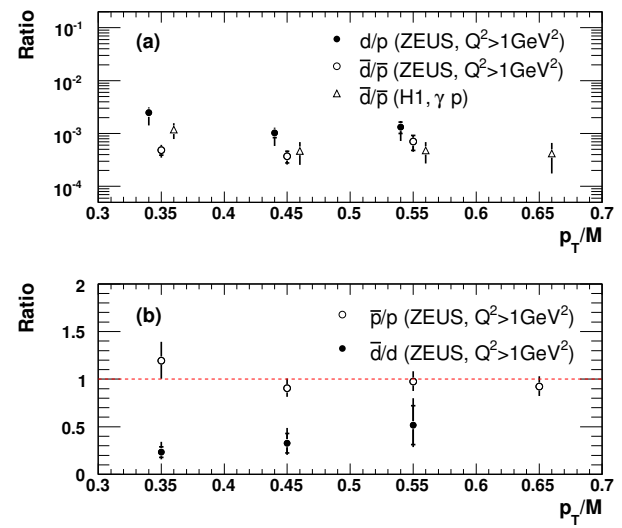

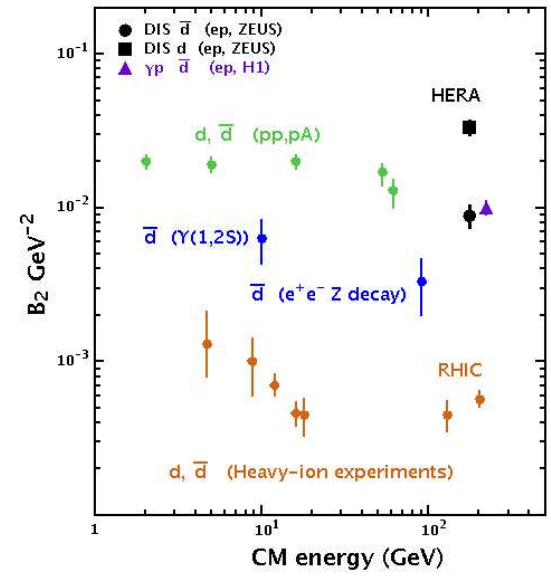

Figure 2. Left: $d / p, \bar{d} / \bar{p}, \bar{p} / p$ and $\bar{d} / d$ production ratios as a function of $p_{T} / M$. Right: Comparison of the $B_{2}$ values extracted from DIS with other world measurements.

The detector-corrected production ratios as a function of $p_{T} / M$ are shown in Fig. 2(left). For the antiparticle ratio, there is a good agreement with the H1 published data for photoproduction [8], as well as with $p p$ data [7]. The production rate of $d$ is higher than that of $\bar{d}$ at low $p_{T}$. If (anti)deuterons are produced as a result of a coalescence of two (anti)nucleons, then one should expect that the $\bar{d} / d$ ratio is approximately equal to the $(\bar{p} / p)^{2}$ ratio for the same $p_{T}$ per (anti)nucleon, assuming the same source radius for particles and antiparticles. In this case, one should expect $\bar{d} / d \simeq 1$, since the measured $\bar{p} / p$ ratio is consistent with unity. Under the assumption that secondary interactions do not produce an enhancement at $D C A=0$ for the $d$ case, the result would indicate that the relation between $\bar{d} / d$ and $(\bar{p} / p)^{2}$ expected from the coalescence model with the same $B_{2}$ for particles and antiparticles does not hold in the central fragmentation region of $e p$ DIS collisions. In terms of the coalescence model, the $d$ production volume in momentum space is larger than that for $\bar{d}$.

For collisions involving incoming baryon beams, there are several models [11] which predict $p-\bar{p}$ production asymmetry in the central rapidity region. The predicted asymmetry can be as high as $7 \%$ due to the presence of the incoming proton. As shown in Fig. 2(left), the experimental data for $p(\bar{p})$ are not sufficiently precise to confirm such expectations.

There are no predictions for the $d-\bar{d}$ asymmetry. It is possible that theoretical expectations for such compound states are different than those for the $p-\bar{p}$ asymmetry, since $d(\bar{d})$ are not contaminated by a large contribution from the standard baryons produced in quarks and gluon fragmentation.

The production of $d(\bar{d})$ was studied in terms of the coalescence model. The $B_{2}$ values 
extracted in the region $0.3<p_{T} / M<0.7$ are shown in Figure 2(right). While the measurement of $B_{2}$ has larger experimental errors than those in the studies of the production asymmetries, it is still seen that $B_{2}$ for $d$ tends to be higher than that for $\bar{d}$. The values of $B_{2}$ for $\bar{d}$ are in agreement with the measurements in photoproduction [8], but disagree with the $B_{2}$ measured in $e^{+} e^{-}$annihilation [4] at the $Z$ resonance. In contrast to heavy-ion collisions, where $B_{2}$ strongly increases as a function of $p_{T}$ due to an expanding source, the $B_{2}$ measured in $e p$ does not strongly depend on $p_{T} / M[10]$.

In summary, the first observation of (anti)deuterons in ep collisions in the DIS regime is presented. The yield of $d(\bar{d})$ is three orders of magnitude smaller than that of $p(\bar{p})$, which is in broad agreement with other experiments. The production rate of $p$ is consistent with that of $\bar{p}$, while the production rate of $d$ is higher than that for $\bar{d}$ for the same kinematic region.

\section{References}

[1] Slides: http://indico. cern. ch/contributionDisplay. py? contribId=229\&sessionId=6\&conf Id=9499 .

[2] S. Butler, C. Pearson, Phys. Rev. 129, 836 (1963).

[3] M. Karliner, B. R. Webber, JHEP 0412, 045 (2004).

[4] ARGUS Collaboration, H. Albrecht, et al., Phys. Lett. B157, 326 (1985); ARGUS Collaboration, H. Albrecht, et al., Phys. Lett. B236, 102 (1990); OPAL Collaboration, R. Akers, et al., Z. Phys. C67, 203 (1995); ALEPH Collaboration, S. Schael, et al., Phys. Lett. B639, 16 (2006).

[5] G. Gustafson, J. Hakkinen, Z. Phys. C61, 683 (1994).

[6] IHEP-CERN Collaboration, F. Binon, et al., Phys. Lett. B30, 510 (1969); Y. Antipov, et al., Phys. Lett. B34, 164 (1971);

J. Cronin, et al., Phys. Rev. D11, 3105 (1975).

[7] B. Alper, et al., Phys. Lett. B46, 265 (1973); BRITISH-SCANDINAVIAN Collaboration, W. Gibson, et al., Nuovo Cim. Lett. 21, 189 (1978); V. Abramov, et al., Sov. J. Nucl. Phys. 45, 845 (1987).

[8] H1 Collaboration, A. Aktas, et al., Eur. Phys. J. C36, 413 (2004).

[9] M. Aoki, et al., Phys. Rev. Lett. 69, 2345 (1992); NA52 (NEWMASS) Collaboration, G. Appelquist, et al., Phys. Lett. B376, 245 (1996); STAR Collaboration, C. Alper, et al., Phys. Rev. Lett. 87, 262301 (2001); E802 Collaboration, L. Ahle, et al., Phys. Rev. C57, 1416 (1998); NA44 Collaboration, I. Bearden, et al., Eur. Phys. J. C23, 237 (2002); PHENIX Collaboration, S. S. Adler, et al., Phys. Rev. Lett. 94, 122302 (2005).

[10] ZEUS Collaboration, S. Chekanov, et al., (2007). DESY-07-070, hep-ex/0705.3770.

[11] B. Kopeliovich, B. Povh, Z. Phys. C75, 693 (1997);

B. Kopeliovich, B. Povh, Phys. Lett. B446, 321 (1999);

G. Garvey, B. Z. Kopeliovich, B. Povh, Comments Mod. Phys. A2, 47 (2001);

S. Chekanov, Eur. Phys. J. C44, 367 (2005);

F. Bopp, Y. Shabelski, Phys. Atom. Nucl. 68, 2093 (2005). 